Artigo Original

\title{
Funcionamento da via auditiva e da linguagem em gêmeas monozigóticas com características de mutismo seletivo
}

\section{Auditory pathway and language functioning of monozygotic twins with characteristics of selective mutism}

Renata Aparecida Leite ${ }^{1}$, Luciene Stivanin², Christian César Cândido de Oliveira ${ }^{3}$, Caroline Rondina ${ }^{4}$, Carla Gentile Matas ${ }^{5}$, Claudia Inês Scheuer ${ }^{6}$

\section{RESUMO}

Introdução: Estruturas do sistema nervoso central podem sofrer modificações funcionais com estimulação. Objetivo: O objetivo deste estudo foi verificar possíveis modificações nos achados audiológicos após intervenção fonoaudiológica em comunicação e linguagem. Métodos: Gêmeas univitelinas com alteração de linguagem foram submetidas às medidas de imitância acústica, audiometria vocal e potencial evocado auditivo de tronco encefálico antes e após quatro meses de intervenção fonoaudiológica. Resultados: Na primeira avaliação audiológica, uma das crianças apresentou alteração da via auditiva no tronco encefálico à direita associado à perda auditiva condutiva. A outra criança apresentou potencial evocado auditivo de tronco encefálico compatível com perda auditiva condutiva à direita. Na segunda avaliação audiológica, houve melhora no potencial evocado auditivo de tronco encefálico em ambas as crianças. Conclusões: Ocorreram modificações nos potenciais evocados auditivos de tronco encefálico em gêmeas monozigóticas com alterações de linguagem após intervenção fonoaudiológica, podendo estas serem decorrentes de plasticidade neuronal.

Unitermos: Potenciais evocados auditivos, Plasticidade neuronal, Linguagem infantil, Terapia de linguagem

Citação: Leite RA, Stivanin L, Oliveira CCC, Rondina C, Matas CG, Scheuer CI. Funcionamento da via auditiva e da linguagem em gêmeas monozigóticas com características de mutismo seletivo. Rev Neurociencias 2005; 13(2): 074-079.

\section{SUMMARY}

Introduction: Central nervous system structures can suffer modifications after stimulations. Purpose: The aim of this study was to verify possible modifications of audiologic findings after speech-language intervention focusing

Trabalho realizado: Faculdade de Medicina da Universidade de São Paulo

1 - Mestranda pelo Programa de Pós-graduação em Fisiopatologia Experimental da FMUSP. Fonoaudióloga colaboradora do Laboratório de Investigação Fonoaudiológica em Potenciais Evocados Auditivos em Tronco Encefálico

2 - Mestranda pelo Programa de Pós-graduação em Fisiopatologia Experimental da FMUSP. Fonoaudióloga supervisora do Laboratório de Investigação Fonoaudiológica em Linguagem Infantil e Cognição do Curso de Fonoaudiologia da FMUSP

3 - Mestrando pelo Programa de Pós-graduação em Fisiopatologia Experimental da FMUSP. Fonoaudiólogo da equipe de Enfermaria de Álcool e Drogas para Adolescentes do Serviço de Psiquiatria da Infância e Adolescência do Hospital das Clínicas da FMUSP

4 - Fonoaudióloga Especializada em Audiologia Clínica pelo Curso de Fonoaudiologia do Departamento de Fisioterapia, Fonoaudiologia e Terapia Ocupacional da FMUSP

5 - Professora Doutora do Curso de Fonoaudiologia do Departamento de Fisioterapia, Fonoaudiologia e Terapia Ocupacional da FMUSP; Docente do programa de Pós-graduação em Fisiopatologia Experimental da FMUSP

6 - Professora Doutora do Curso de Fonoaudiologia do Departamento de Fisioterapia, Fonoaudiologia e Terapia Ocupacional da FMUSP; Docente do programa de Pós-graduação em Fisiopatologia Experimental da FMUSP 
communication and language. Methods: Two girls, monozygotic twins with language disorder, were submitted to audiologic assessments that included acoustic immitance measures, vocal audiometry and brainstem auditory evoked potential, before and after four months of speech-language intervention. Results: In the first audiologic evaluation, one of the twins presented abnormal brainstem auditory pathway at the right, associated to conductive hearing loss. The other twin had auditory brainstem evoked potential compatible with right conductive hearing loss. In the second assessment, both children showed improvements on the brainstem auditory evoked potential. Conclusions: It was observed modifications on the brainstem auditory evoked potentials of monozygotic twins with language disorder after speech-language intervention, possibly caused by neuronal plasticity.

\section{Keywords: Auditory evoked potentials, Neuronal plasticity, Child language, Language therapy}

Citation: Leite RA, Stivanin L, Oliveira CCC, Rondina C, Matas CG, Scheuer Cl. Auditory pathway and language functioning of monozygotic twins with characteristics of selective mutism. Rev Neurociencias 2005; 13(2): 074-079.

\section{INTRODUÇÃO}

O Mutismo Seletivo está inserido nos distúrbios psiquiátricos que afetam amplamente o desenvolvimento, sendo geralmente diagnosticado pela primeira vez na infância ou adolescência. Os critérios diagnósticos são: fracasso persistente em falar em situações sociais específicas (nas quais existe a expectativa para falar, por ex. na escola), apesar de falar em outras situações; a perturbação interfere na realização educacional ou na comunicação social; a duração da perturbação é de, no mínimo, um mês (não limitada ao primeiro mês de escolarização); o fracasso em falar não é devido a uma falta de conhecimento ou desconforto com a linguagem falada exigida pela situação social; a perturbação não é explicada como um Transtorno da Comunicação, nem decorrente exclusivamente durante o curso de um Transtorno Invasivo do Desenvolvimento, Esquizofrenia ou outro Transtorno Psicótico ${ }^{1}$.

Atualmente, o Mutismo Seletivo (MS) é considerado uma patologia de etiologia multifatorial que pode vir associada a transtornos de ansiedade e déficit de linguagem², em áreas como expressão verbal e compreensão.

Na literatura consultada, não foram encontrados estudos sobre desenvolvimento de linguagem em gêmeos com mutismo seletivo. Sabe-se, porém, que crianças gêmeas podem apresentar atraso no desenvolvimento de linguagem. Este atraso é determinado pela maneira peculiar de viver unido a um irmão (situação gemelar), fato que não produz nenhuma pressão ou necessidade de comunicação verbal. Essa relação particular pode levar a uma persistência na imaturidade do desenvolvimento de linguagem e global, mais do que produzir padrões de fala com desvio ${ }^{3-5}$.

Estudos em indivíduos com alterações de linguagem sugerem que a falha na codificação neural da informação auditiva parece ter um papel importante no desenvolvimento das habilidades de linguagem, provavelmente em função da alteração na percepção de pistas acústicas importantes contidas nos sinais de fala ${ }^{6}$.

De acordo com a literatura consultada, dificuldades na percepção dos sons da fala ocorrem por uma falha na representação do sinal da fala nos centros auditivos cen- trais, decorrentes de deficiência na sincronia neural. Tais dificuldades podem ser melhoradas e modificadas por meio da aprendizagem perceptual associada ao treinamento auditivo ${ }^{7}$.

Esta possibilidade de modificação, adaptabilidade, nova morfologia, nova funcionalidade ou ainda capacidade de transformação ocorrida após estimulação, devese à plasticidade neuronal, termo aplicável em situações onde determinadas estruturas se modificam de forma adaptativa às exigências de desempenho que pode ser auditivo, cognitivo, motor, visual, entre outros ${ }^{8}$.

Estas modificações no substrato neural podem ocorrer devido à prática de uma habilidade ou exposição freqüente a um estímulo9,10.

Uma das formas de avaliar a plasticidade neuronal é por meio dos potenciais evocados auditivos ${ }^{8}$. O potencial evocado auditivo mais utilizado na prática clínica é o Potencial Evocado Auditivo de Tronco Encefálico (PEATE), que é um exame objetivo da audição utilizado na avaliação da via auditiva desde o nervo auditivo até o tronco encefálico, que complementa os procedimentos audiológicos de rotina no diagnóstico de desordens auditivas $^{11}$

Sabendo-se da importância do funcionamento adequado da via auditiva para o desenvolvimento da linguagem, é fundamental avaliar crianças que apresentam alterações de linguagem.

Dessa forma, o objetivo deste estudo foi verificar possíveis modificações nos achados audiológicos após intervenção fonoaudiológica em comunicação e linguagem em gêmeas monozigóticas com alterações de linguagem.

\section{MATERIAL E MÉTODO}

Participaram deste estudo de caso gêmeas monozigóticas com idade de cinco anos. As crianças compareceram ao serviço de Audiologia do Hospital Universitário da Universidade de São Paulo no primeiro semestre de 2003, encaminhadas pelo otorrinolaringologista do próprio hospital por suspeita de otite. 
Diante da dificuldade de interação apresentada pelas crianças, estas foram encaminhadas para avaliação de linguagem no Laboratório de Investigação Fonoaudiológica em Linguagem Infantil e Cognição e para avaliação audiológica no Laboratório de Investigação Fonoaudiológica em Potenciais Evocados Auditivos do Curso de Fonoaudiologia da Faculdade de Medicina da Universidade de São Paulo.

Antes de iniciar a avaliação, o responsável pelas crianças assinou um termo de consentimento livre e esclarecido, no qual estavam descritos todos os procedimentos a serem realizados. Este trabalho foi aprovado pela Comissão de Pesquisa do Departamento de Fisioterapia, Fonoaudiologia e Terapia Ocupacional da Faculdade de Medicina da Universidade de São Paulo, sob Protocolo no. 037/2005.

Embora a intenção de procurar o serviço não tenha sido da mãe, esta manifestou sua queixa em relação ao desenvolvimento das crianças: fala ininteligível e ausência de comunicação em determinadas situações, e com determinados interlocutores.

De acordo com os dados de anamnese, não ocorreram alterações pré, peri e pós-natais e não houve atraso no desenvolvimento neuropsicomotor. A aquisição de linguagem foi tardia. Atualmente, ambas as crianças compreendem gestos mas não os utilizam, e comunicam suas necessidades por meio de linguagem oral, caracterizada por falta de inteligibilidade de fala. Quanto à questão social, estas apresentam comportamento retraído em ambientes públicos, não se relacionam com pessoas que não são da família e dificilmente fazem amizades. As crianças freqüentam ensino infantil (pré-escola); apresentam dificuldades de adaptação e a história clínica é de otites de repetição, problemas respiratórios, além de histórico familiar para alterações comportamentais e psiquiátricas.

A linguagem foi avaliada informalmente com ambas as crianças juntas, em observação de jogo espontâneo, para verificar o comportamento, questões cognitivas, comunicação e linguagem. Os resultados da avaliação de linguagem mostraram que houve realização de atividade simbólica, com ocorrência de ações complementares e ação compartilhada. A comunicação entre as gêmeas foi por meio de olhares, expressões faciais e gestos, sem fala ou oralidade. Observou-se também que não se dirigiram aos avaliadores e quando, por alguma necessidade, escoIheram ou selecionaram apenas um deles para se comunicar. Ambas as meninas apresentavam muitas dificuldades para contatar ou comunicar-se com os outros interlocutores, mostrando forte resistência na interação com pessoas do sexo masculino. Contudo, mostraram-se muito atentas às ações e à comunicação entre si mesmas, excluindo estímulos ambientais.

A avaliação audiológica constou de visualização do meato acústico externo realizada com o otoscópio da marca HEINE, medidas de imitância acústica obtidas no imitanciômetro modelo GSI-33 marca GrasonStandler, audiometria tonal e vocal realizadas com o Audiômetro GSI 68 - marca Grason-Stadler e PEATE utilizando o equipamento portátil Modelo Traveler-Express da marca Biologic.

Para a realização do PEATE foi realizada inicialmente a limpeza da pele com pasta abrasiva, sendo os eletrodos fixados à pele do indivíduo por meio de pasta eletrolítica e fita adesiva (micropore), nas seguintes posições: vértex $(\mathrm{Cz})$ e mastóides direita e esquerda (A2 e A1). Os valores de impedância dos eletrodos foram verificados, situando-se abaixo de 5 Kohms. O estímulo acústico utilizado foi o clique de polaridade rarefeita apresentado monoauralmente por um par de fones TDH-39 a 80 dB NA, em uma velocidade de apresentação de 19,0 cliques por segundo, sendo empregado um total de 2000 estímulos. Foram utilizados filtros passa-baixo e passa-alto de 100 e $3000 \mathrm{~Hz}$ respectivamente para registro das respostas.

As crianças foram encaminhadas para avaliação e conduta otorrinolaringológicas. Durante o atendimento audiológico, as crianças estiveram em companhia dos terapeutas devido à dificuldade do estabelecimento de vínculo.

Após as avaliações iniciais de linguagem e audiológica, as crianças foram submetidas à intervenção fonoaudiológica em comunicação e linguagem.

O objetivo da intervenção fonoaudiológica foi trabaIhar habilidades cognitivas relacionadas à atenção e memória, ao mesmo tempo estimulando melhor qualidade na interação e maior produção da linguagem oral, por meio de atividades lúdicas. As crianças foram atendidas conjuntamente, em função das dificuldades para realizarem atividades separadas, em sessões realizadas por dois terapeutas.

Após um período de quatro meses de intervenção fonoaudiológica, o funcionamento da via auditiva foi reavaliado utilizando os mesmos procedimentos empregados na primeira avaliação audiológica.

\section{RESULTADOS}

Com relação às avaliações audiológicas antes e após intervenção fonoaudiológica obteve-se os seguintes resultados:

\section{Primeira avaliação audiológica (antes da inter- venção fonoaudiológica)}

Caso 1 (C1): obteve-se nas medidas de imitância acústica curva timpanométrica tipo B à direita e tipo C à esquerda. Não foi possível a realização da 
audiometria tonal devido à dificuldade de estabelecimento de vínculo da criança com a avaliadora, fato que dificultou também o condicionamento lúdico para que pudessem responder ao exame. Realizou-se Audiometria Vocal, com Limiar de Recepção de Fala (LRF) obtido por meio de ordens simples, apresentando resultados compatíveis com perda auditiva de grau moderado à direita $(L R F=45 \mathrm{~dB})$ e leve à esquerda $(L R F=20 \mathrm{~dB})$. Frente ao comprometimento de orelha média não foi possível a realização das emissões otoacústicas. No resultado do potencial evocado auditivo de tronco encefálico verificou-se presença das Ondas I, III, V a 80 dB NA para cliques bilateralmente, com latências absolutas normais (Onda I = 1,48 milissegundos - $\mathrm{ms}$; Onda III= 3,52 ms; Onda $V=5,60 \mathrm{~ms}$ ) e interpicos I-III, III-V, I-V normais (Interpico I-III= 2,04 ms; Interpico III-V= 2,08 ms; Interpico $\mathrm{I}-\mathrm{V}=4,12 \mathrm{~ms}$ ) à esquerda, e aumento nas latências absolutas das Ondas I, III, V (Onda I= 2,12 ms; Onda III=4,12 ms; Onda $V=7,48 \mathrm{~ms}$ ) e nos interpicos III-V e I-V (Interpico III-V= 3,36 ms e Interpico $\mathrm{I}-\mathrm{V}=5,36 \mathrm{~ms}$ ) à direita, sugerindo alteração na via auditiva em tronco encefálico alto à direita associado à perda auditiva condutiva (Figura 1).

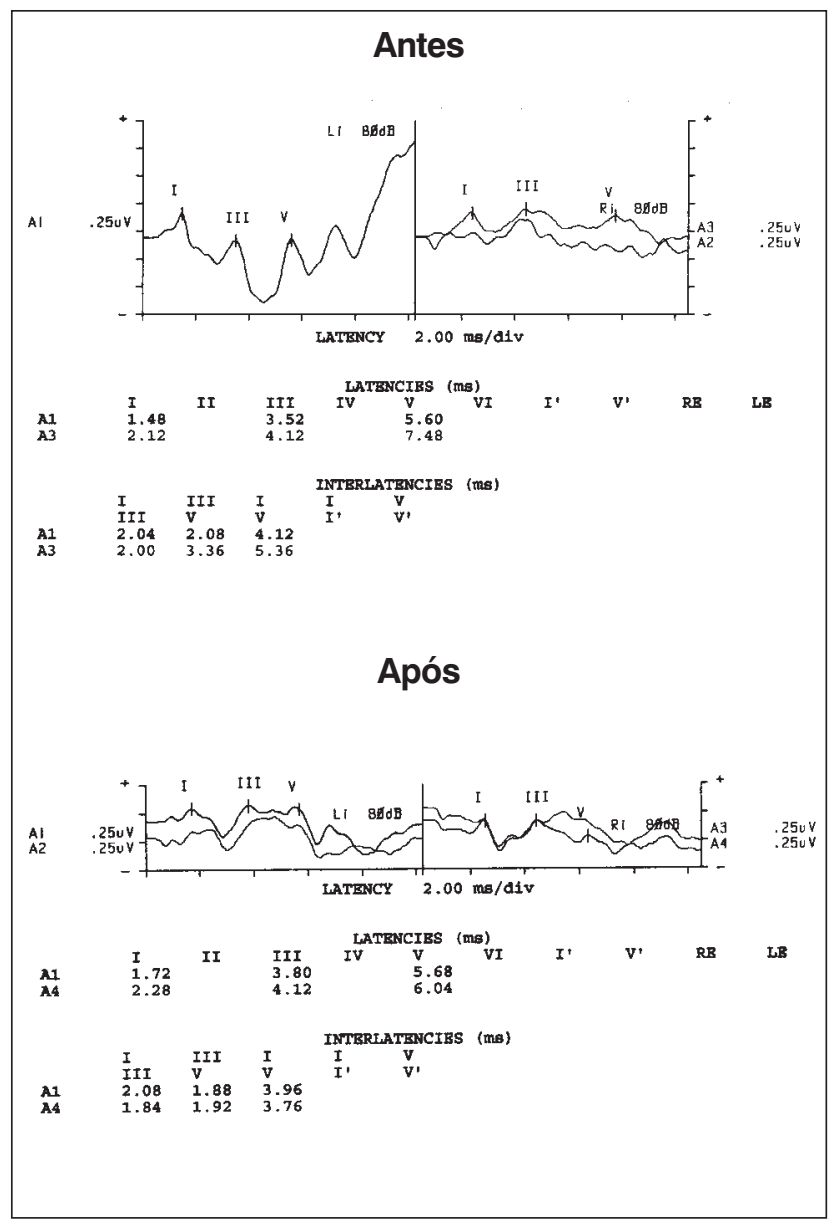

Figura 1. Valores das latências absolutas das Ondas I, III, V e interpicos I-III, III-V, I-V de C1, obtidos no PEATE antes e após intervenção fonoaudiológica.
Caso 2 (C2): obteve-se nas medidas de imitância acústica curva timpanométrica curva tipo B à esquerda, não sendo avaliada a orelha direita devido à perfuração de membrana timpânica. Não foi possível a realização da audiometria tonal condicionada devido à dificuldade de estabelecimento de vínculo da criança com a avaliadora. Foi realizada Audiometria Vocal, com Limiar de Recepção de Fala (LRF) obtido por meio de ordens simples, apresentando resultados compatíveis com perda auditiva de grau leve bilateralmente $(\mathrm{LRF}=35 \mathrm{~dB}$ orelha direita e $40 \mathrm{~dB}$ orelha esquerda). Devido ao comprometimento de orelha média não foi realizado o teste de emissões otoacústicas. No potencial evocado auditivo de tronco encefálico observou-se presença das Ondas I, III, V a 80 dB NA para cliques bilateralmente, com latências absolutas normais (Onda I= $1,64 \mathrm{~ms}$; Onda III=3,48 ms; Onda $V=5,56 \mathrm{~ms}$ ) e interpicos I-III, III-V, I-V normais (Interpico I-III = 1,84 ms; Interpico III-V= $2,08 \mathrm{~ms}$; Interpico I-V=3,92 ms) à esquerda, e com latências absolutas levemente aumentadas (Onda I= 1,88 ms; Onda $\mathrm{III}=3,76 \mathrm{~ms}$; Onda $\mathrm{V}=5,84 \mathrm{~ms}$ ) e interpicos normais (Interpico I-III = 1,88 ms, Interpico III-V=2,08 ms, Interpico I$\mathrm{V}=3,96 \mathrm{~ms}$ ) à direita, compatível com perda auditiva condutiva e sem evidências de comprometimento da via auditiva no tronco encefálico bilateralmente (Figura 2).

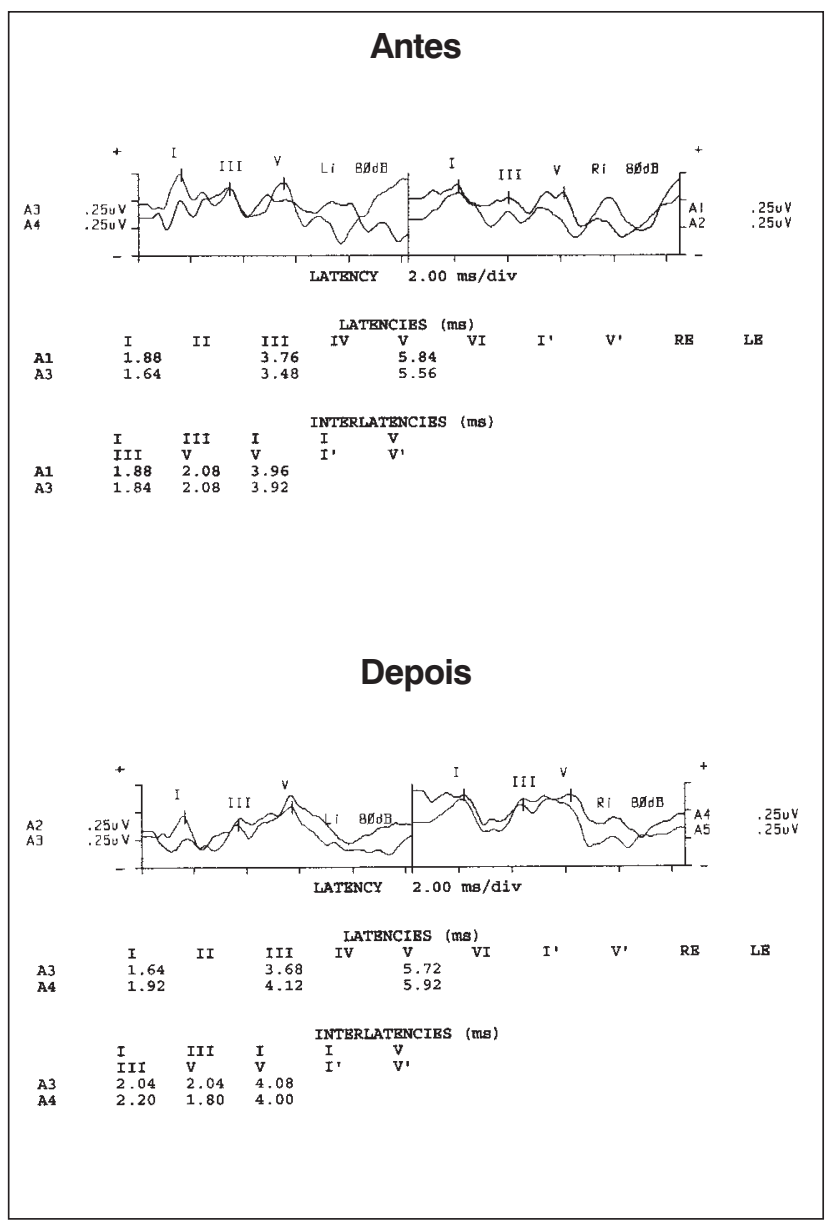

Figura 2. Valores das latências absolutas das Ondas I, III, V e interpicos I-III, III-V, I-V de C2, obtidos no PEATE antes e após intervenção fonoaudiológica. 


\section{Segunda avaliação audiológica (após interven- ção fonoaudiológica)}

Caso 1 (C1): nas medidas de imitância acústica observou-se curva timpanométrica tipo $\mathrm{B}$ com reflexos acústicos ausentes ipsi e contralateralmente em ambas as orelhas. Novamente a criança não colaborou para a realização da audiometria tonal, sendo realizado apenas o LRF por meio de ordens simples, mantendo os resultados obtidos anteriormente: perda auditiva de grau moderado à direita $(L R F=45 \mathrm{~dB})$ e leve à esquerda $(\mathrm{LRF}=20 \mathrm{~dB})$. Verificou-se nos potenciais evocados auditivos melhora nos resultados à direita, com diminuição significativa da latência absoluta da Onda V (Onda $\mathrm{V}=6,04 \mathrm{~ms}$ ) e interpicos III-V e I-V (Interpico III-V=1,92; Interpico $\mathrm{I}-\mathrm{V}=3,76 \mathrm{~ms}$ ), quando comparadas com os resultados obtidos na primeira avaliação (Figura 1). Tais dados foram compatíveis com perda auditiva condutiva mais acentuada à direita, não sendo mais evidenciado comprometimento da via auditiva no tronco encefálico.

Caso 2 (C2): nas medidas de imitância acústica observou-se curva timpanométrica tipo B com reflexos acústicos ipsi e contralaterais ausentes bilateralmente. C2 também não colaborou para a realização da audiometria tonal condicionada, realizando somente o LRF por meio de ordens simples, mantendo os resultados obtidos anteriormente: perda auditiva de grau leve bilateralmente (LRF= $35 \mathrm{~dB}$ orelha direita e $40 \mathrm{~dB}$ orelha esquerda). Observou-se nos potenciais evocados auditivos de tronco encefálico resultados semelhantes aos obtidos na primeira avaliação, porém com uma melhora significativa na morfologia das ondas após intervenção fonoaudiológica (Figura 2).

Os resultados obtidos nos Potenciais Evocados Auditivos de Tronco Encefálico dos casos 1 e 2, antes e após intervenção fonoaudiológica, encontram-se descritos no Quadro 1.

\section{DISCUSSÃO}

Após o início da intervenção fonoaudiológica foram observadas mudanças na comunicação, no comportamento e funcionamento cognitivo de ambas as gêmeas, bem como no potencial evocado auditivo de tronco encefálico.

Em relação à linguagem, foi difícil caracterizar o comportamento de cada criança separadamente, pois estas alternavam o comportamento durante as terapias e porque selecionavam o interlocutor. Porém, houve meIhora significativa na comunicação interpessoal, com aumento da produção oral e melhora na inteligibilidade de fala, no contexto terapêutico e social.

Entretanto, no potencial evocado auditivo de tronco encefálico, um teste objetivo, pôde-se caracterizar a via auditiva de cada criança separadamente. Observou-se na primeira avaliação audiológica de uma das crianças, alteração da via auditiva no tronco encefálico alto sendo que na reavaliação, após intervenção fonoaudiológica, houve uma melhora nos resultados, não sendo mais evidenciado comprometimento nesta porção da via auditiva, além de ter ocorrido uma melhora significativa do traçado. Em relação à outra criança, não foram observadas alterações da via auditiva no tronco encefálico na primeira avaliação, observando-se na reavaliação meIhora significativa na morfologia das ondas após intervenção fonoaudiológica.

A estimulação freqüente das habilidades cognitivas e de linguagem durante a intervenção fonoaudiológica parece ter ocasionado modificações no processamento neural da informação auditiva. De acordo com a literatura, determinadas estruturas modificam-se de forma adaptativa após estimulação devido à plasticidade neuronal ${ }^{8-10}$. Este fato pode ser comprovado por exames de imagem, nos quais se observam modificações na atividade cerebral após breve período de estimulação ${ }^{12}$. Segundo a literatura especializada, tais modificações também podem ser observadas por meio dos potenciais evocados auditivos $^{8}$, incluindo, portanto, o potencial evocado auditivo de tronco encefálico.

Neste estudo, a melhora constatada na reavaliação audiológica, após quatro meses de intervenção fonoaudiológica, sugere que a intervenção enfocada na comunicação, linguagem e cognição ocasionou uma

\begin{tabular}{|c|c|c|c|c|c|c|c|c|c|c|c|c|c|}
\hline & & \multicolumn{6}{|c|}{ Antes da intervenção fonoaudiológica } & \multicolumn{6}{|c|}{ Após intervenção fonoaudiológica } \\
\hline & & Onda I & $\begin{array}{l}\text { Onda } \\
\text { III }\end{array}$ & $\begin{array}{l}\text { Onda } \\
\text { v }\end{array}$ & $\begin{array}{l}\text { Interpico } \\
\qquad \text { |-I| }\end{array}$ & $\begin{array}{l}\text { Interpico } \\
\qquad I \mid-V\end{array}$ & $\begin{array}{c}\text { Interpico } \\
\text { I-V }\end{array}$ & Onda I & $\begin{array}{l}\text { Onda } \\
\text { III }\end{array}$ & $\begin{array}{l}\text { Onda } \\
\mathrm{V}\end{array}$ & $\begin{array}{l}\text { Interpico } \\
\text { |-I|| }\end{array}$ & $\begin{array}{c}\text { Interpico } \\
\text { III-V }\end{array}$ & $\begin{array}{c}\text { Interpico } \\
\text { I-V }\end{array}$ \\
\hline Caso & OD & 2,12 & 4,12 & 7,48 & 2,00 & 3,36 & 5,36 & 2,28 & 4,12 & 6,04 & 1,84 & 1,92 & 3,76 \\
\hline 1 & OE & 1,48 & 3,52 & 5,60 & 2,04 & 2,08 & 4,12 & 1,72 & 3,80 & 5,68 & 2,08 & 1,88 & 3,96 \\
\hline Caso & OD & 1,88 & 3,76 & 5,84 & 1,88 & 2,08 & 3,96 & 1,92 & 4,12 & 5,92 & 2,20 & 1,80 & 4,00 \\
\hline 2 & OE & 1,64 & 3,48 & 5,56 & 1,84 & 2,08 & 3,92 & 1,64 & 3,68 & 5,72 & 2,04 & 2,04 & 4,08 \\
\hline
\end{tabular}

Quadro 1 - Resultados (em milissegundos) obtidos nos Potenciais Evocados Auditivos de Tronco Encefálico dos casos 1 e 2, antes e após intervenção fonoaudiológica. 
adaptação ou modificação na organização estrutural, bem como no funcionamento do Sistema Nervoso Central especificamente no tronco encefálico, levando a uma melhora na sincronia neural nesta região.

Portanto, compreender o funcionamento do sistema auditivo central e de sua capacidade de plasticidade neuronal é de extrema importância para o conhecimento de como o cérebro integra e discrimina os estímulos mais complexos vindos do meio, incluindo os sons da fala. Conhecer os mecanismos pelos quais as estruturas centrais do sistema auditivo reagem a uma nova entrada de estímulos pode auxiliar no desenvolvimento de métodos que garantam um melhor aproveitamento para a comunicação do indivíduo.

A intervenção fonoaudiológica pode ter favorecido a plasticidade neuronal, refletida por mudanças na comunicação e no potencial evocado auditivo de tronco encefálico nas gêmeas com acentuadas dificuldades comunicativas, comportamentais e cognitivas. No entanto, o que não foi objeto deste estudo e que deve ser no futuro, é verificar se mudanças objetivas em testes audiológicos que envolvem o Sistema Nervoso Central, levam a novas mudanças e melhoras na comunicação e linguagem do indivíduo.

\section{CONCLUSÃO}

Houve modificações nos potenciais evocados auditivos de tronco encefálico após intervenção fonoaudiológica em gêmeas monozigóticas com alterações de linguagem.

\section{REFERÊNCIAS BIBLIOGRÁFICAS}

1. DSM-IV, Diagnostic And Statistical Manual Of Mental Disorders. $4^{\text {nd }}$ ed. Washington: Copyright. American Psychiatric Association, 1994

2. Kristensen $\mathrm{H}$, Torgersen S. MCMI-II personality traits and symptom traits in parenes of children with selective mutism: a case-control study. J Abnorm Psychol 2001; 110:648-652.

3. Dodd B, Mcevoy S. The communication abilities of 2-to-4years-old twins. Eur J Disord Commun 1994; 21:273-289.

4. Bishop DVM, Bishop SI. Twin Language: A risk factor for language impairment. J. Speech Lang Hear Res 1998; 41: 150-160.

5. Thorpe K, Rutter M, Greenwood R. Twins as a natural experiment to study the causes of mild language delay. II: Family Interaction Risk Factors. J Child Psychol \& Psychiat 2003; 44:342-355.

6. Wible B, Nicol T, Kraus N. Correlation between brainstem and cortical auditory processes in normal and languageimpaired children. Brain 2005; 128(2): 417-423.
7. Kraus N. Auditory pathway encoding and neural plasticity in children with learning problems. Audiol. Neurootol.2001;6:221-227.

8. Kraus N. Speech sound perception, neurophysiology, and plasticity. Int J Pediatr Otorhinolaryngol 1999; 47:123-129.

9. Grafman J. Conceptualizing Functional Neuroplasticity J Commun Disord 2000; 33:345-356.

10. Karni A. Adult cortical plasticity and reorganization. Sci Méd 1997; 24-33

11. Matas CG, Frazza MM, Munhoz MLL. Aplicação do potencial auditivo de tronco encefálico em audiologia pediátrica. In: Basseto MCA, Brock R, Wajnsztejn R (eds). Neonatologia. Um convite à atuação Fonoaudiológica. São Paulo: Editora Lovise, 1998. p 301-310.

12. Posner Ml. Imaging a Science of mind. Trends Cogn Sci 2003; 7(10): 450-453. 